\title{
Insights from next generation sequencing of the cancer genome
}

\author{
M Porteous \\ Consultant Clinical Geneticist, NHS Lothian, and Honorary Professor, University of Edinburgh, Edinburgh UK
}

TITLE Exploring the genomes of cancer cells: progress and promise

AUTHORS MR Stratton

JOURNAL Science 20 I I; 33 I:I553-58. http://dx.doi.org/I0.I I 26/science. 1204040

DECLARATION OF INTERESTS No conflicts of interest declared.

\author{
Correspondence to M Porteous, \\ SE Scotland Genetic Service, \\ Western General Hospital, \\ Crewe Road, Edinburgh \\ EH4 2XU, UK
}

tel. +44 (0) I3I 537 I093

e-mail mary.porteous@ed.ac.uk

\section{SUMMARY}

This detailed review provides a history and update of the complex field of cancer genetics. Using examples of progress such as the development of imatinib for chronic myeloid leukemia (which stemmed from the recognition of the Philadelphia chromosome in the 1960s), Michael Stratton, Director of the Sanger Institute in Cambridge is confident that we are now entering the end game of cancer genome sequencing. This has led to other drug therapies, based on knowledge of the molecular changes in some cancers, lung and breast cancers for example.

About 400 human protein coding genes contribute to neoplastic change. Of these, $80 \%$ are dominantly acting, partly due to ascertainment bias. Stratton distinguishes two types of mutation, driver and passenger mutations. The former progress tumour growth and spread; passenger mutations do not do this, but they can be used to record the history of cancer cells in individuals. Thus, molecular changes in the cancer genome provide an 'archeological record' of the mutagenic processes (drivers and passengers) that led to the cancer. The molecular patterns also link some sporadic mutations with those caused by, for example, ultraviolet light exposure in melanoma, tobacco carcinogens in lung cancer or aflatoxins in hepatocellular cancer. It is also possible to look at the timing of mutations. For many cancers, mutations seem to accumulate steadily over time; for others there may be sudden, catastrophic molecular event, perhaps during a single cell cycle. The genetic signature of the cells in metastases may show that these develop from a single clone in individual organs. For example, in pancreatic cancer, metastases to the lung may have similar molecular signatures, but these are different from those seeding in the abdominal cavity.

\section{OPINION}

High-throughput genetic sequencing technologies along with DNA sequence information from multiple populations has opened up a major new area of cancer research.This elegant review documents recent advances in our understanding of the molecular basis of cancer. Stratton, who also contributes to the International Cancer Genome Consortium (ICGC), brings a practical medical perspective to the field, still dominated by bioinformatics. Attention may now shift to understanding human genetic variation in health and disease. It is easy to become cynical about the exaggerated claims being made and vast sums of money being consumed by 'big science' programmes. However this review shows how well-designed projects can offer real insights into how the human genome functions.

But what about the 'big leap forward', the dawn of 'personalised medicine'? We are not there yet but the work of the ICGC is exciting. We are used to monitoring remission in haematological malignancies by looking for cells carrying characteristic disease-associated chromosome translocations or by seeking, for example, $B C R-A B L$ mutations by polymerase chain reaction methods. This approach is now technically feasible by looking for very low levels of particular DNA mutations from solid tumour cells that have died, thus leaking their DNA into the patient's bloodstream. Stratton even suggests that such an approach could facilitate detection of cancers before they become symptomatic.

With continuing strong collaboration between academia and service providers and with more clear evidencebased protocols for diagnostic testing established, such as the many examples in this review, the future looks bright. 